\title{
Efficient Downlink Cooperative Multi-cell Transmission for MU-MIMO System
}

\author{
Zhijia Liu, Zesong Fei and Jingming Kuang \\ School of Information and Electronics \\ Beijing Institute of Technology \\ Beijing, China \\ Email: \{111783, feizesong\}@bit.edu.cn
}

\begin{abstract}
Cooperative communication technology has been widely studied in recent years due to providing higher resource utilization and more reliable communication. In this work, we consider a downlink multi-user multi-input-multi-output (MUMIMO) system with cooperative multi-cell transmission. Assuming that the channel state information are available at cell and user, we propose a simple user scheduling algorithm which can achieve the multi-user diversity gain and a power allocation scheme that subject to the total transmission power constrains so that it can more efficiently utilize the system resource and suppress the co-channel interference for the target user equipment. Theoretical analysis and Monte Carlo simulation results show that the transmission sum-rate of the proposed scheme is higher than the traditional single-cell or multi-cell transmission for single user. Additionally, performance of the edge user will also be improved when using the proposed scheme.
\end{abstract}

Keywords-Cooperative multi-cell; MU-MIMO; scheduling; maximal-ratio-combining (MRC)

\section{INTRODUCTION}

In the conventional wireless cellular multi-user MIMO system, signal processing is performed on a per-cell basis. At the same time, the growing demand for ubiquitous access to high-data rate services put huge pressure in the next wireless communication system, such as IMT-Advanced, LTEAdvanced and so on. They all set the goal as providing peak rate of downlink and uplink with $1 \mathrm{Gbps}$ and 500Mbps respectively. However, the traditional single cell transmission system can't satisfy this goal. What's worse, the performance of the edge UE will be restricted largely in the single cell system due to the serious fading and co-channel interference so that the performance of the edge UE can't be guaranteed. To combat the co-channel interference for the edge UE, many preprocessing methods are used, such as precoding and decoding at transmitters and receivers respectively. It is well known that dirty paper coding (DPC) achieves the capacity limitation of the MIMO broadcast channel [1], and many nonlinear precoding methods can provide excellent performance at the cost of the high complexity. However, several linear precoding methods also can get sub-optimal performance. Many joint or independent precoding schemes, such as zeroforcing (ZF), BD, space-time-block-code (STBC), channel inversion and maximum signal-leakage-noise-ratio $\left(\mathrm{SLNR}_{\max }\right)$, have already been investigated in the literature for single user

This work was sponsored by Ericsson.

Corresponding author: Zesong Fei, feizesong@bit.edu.cn or multiple user case [2-6]. Many diversity techniques are discussed and deployed to solve the fading problem, such as time, frequency and spatial diversity. Recently, a new cooperative technology that coordinated multi-cell transmission was proposed for improving the system resource utilization rate and achieving higher signal rate. The idea of coordinated multi cell transmission has been proposed in $[7,8]$ to mitigate the interference and enhance the performance of the edge user. A cooperation system among base station is investigated with slow fading channel [9], and three levels of BS coordination are considered. A multi-cell cooperative ZFBF scheme is presented in [10], combined with a simple sub-optimal user selection procedure for the Wyner downlink channel setup. Many advantages of the multi BS cooperation are introduced [11], such as the capability for co-channel rank/conditioning, macro-diversity and so on. In particular, it provides an upper bound for the achievable performance with BS cooperation at the cost of significant system overhead.

In [12], from the UE's signal-interference-noise-ratio (SINR) constrains perspective, a novel algorithm is contributed, which is capable of efficiently finding the globally optimal downlink beamforming vector across all base station. And, reference [13] gives a joint design of transmit and receive beamforming to weighted sum-rate maximization subject to per BS power constraints. From both information-theoretic and practical signal-processing cells of view, we propose a downlink cooperative multi-cell transmission network with simple user scheduling and power allocation algorithm for the improvement of both performance of the edge UE and system resource utilization.

The rest of the paper is organized as follows. In section II, we describe the system model. This is followed by a simple user scheduling algorithm in section III A, then the process of non-coherent coordinated transmission will be illustrated in section III B. And, the power allocation and system rate will be analyzed in section III C. Numerical simulation and analysis are given in section IV. Finally, conclusions are drawn in section $\mathrm{V}$.

It should be noted that in this paper, we express the matrix or vector using bold type. The superscripts in $\mathbf{G}^{\boldsymbol{T}}, \mathbf{G}^{-1}$ and $\mathbf{G}^{\boldsymbol{H}}$ denote respectively, the transpose, inversion and the conjugate transpose. And $\|\mathbf{G}\|_{F}$ denote the Frobenius-Norm of the matrix G. Then, $\mathbf{I}_{M}$ denote the $M \times M$ identity matrix. 


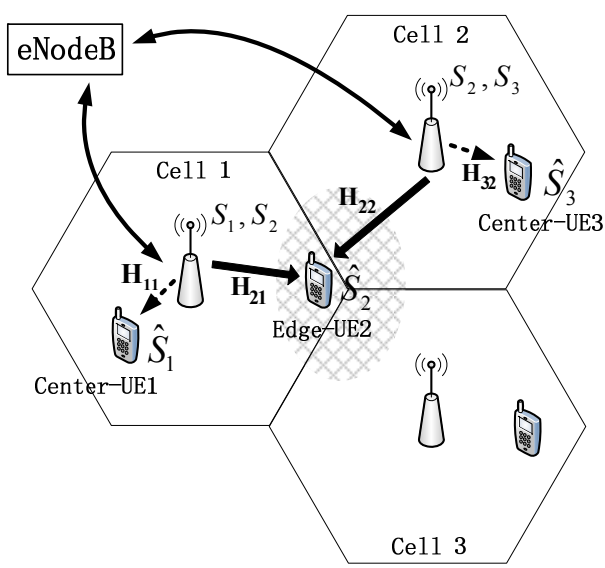

Figure 1. Downlink Cooperative Multi-cell Transmission System Model

\section{SYSTEM MODEL}

We consider a downlink cooperative multi-cell MU-MIMO system with one eNodeB, 2 cells, and $K$ UEs as illustrated in Fig. 1. As generally known, the eNodeB is a combination of the NodeB and RNC that has the function of scheduling and radio resource management. Each cell and UE is equipped with $N_{t}$ and $N_{r}$ antennas respectively. Assume that the perfect CSI is available at the transmitter and receiver. Furthermore, in this paper, we suppose the transmission time frame for sending message is split into two. In the first phase, the two cells select one suitable edge-UE and two center-UEs based on the channel matrix Frobenius-Norm throughout the eNodeB operation. And in the second phase, these two cells transmit the desired signal to the target UE set. Note that the eNodeB supervises and manages all the processing in the system. Lastly, the target UE set contains an edge UE which is served by two cells jointly, and two center UEs each of which is served by only one cell. $\mathbf{S}=\left[S_{1}, S_{2}, S_{3}\right]$ is the transmitted signal vector. $S_{2}$ is the desired signal for edge UE2, and $S_{1}, S_{3}$ are the desired signal for center UE1 and center UE3 respectively. We assume that the channel is the flat Rayleigh fading channel, and the elements of each user's channel matrix is independent complex Gaussian random variable with zero mean and unit variance. The channel between the $j$ th cell and the $i$ th UE is represented by an $N_{r} \times N_{t}$ matrix $\mathbf{H}_{\mathbf{i j}}$. And, $\mathbf{n}_{\mathbf{i}}$ is the additive white Gaussian noise (AWGN) vector of size $N_{r} \times 1$ observed at the receiver. Therefore, the signal processing procedure is given by Equation (1), where $\mathbf{y}_{\mathbf{i}}$ is the received signal vector of the $i$ th UE, $\mathbf{f}_{\mathbf{i}}^{\mathbf{j}}$ with $N_{t} \times 1$ and $p_{i j}$ denote the weighted precoding vector and the transmitted power for the $i$ th UE in the $j$ th cell respectively.

$$
\left[\begin{array}{l}
\mathbf{y}_{1} \\
\mathbf{y}_{\mathbf{2}} \\
\mathbf{y}_{3}
\end{array}\right]=\left[\begin{array}{cc}
\mathbf{H}_{11} & \mathbf{0} \\
\mathbf{H}_{21} & \mathbf{H}_{22} \\
\mathbf{0} & \mathbf{H}_{32}
\end{array}\right]\left[\begin{array}{cccc}
\mathbf{f}_{1}^{1} & \mathbf{f}_{2}^{\mathbf{1}} & \mathbf{0} & \mathbf{0} \\
\mathbf{0} & \mathbf{0} & \mathbf{f}_{2}^{2} & \mathbf{f}_{3}^{2}
\end{array}\right]\left[\begin{array}{cccc}
\sqrt{p_{11}} & 0 & 0 & 0 \\
0 & \sqrt{p_{21}} & 0 & 0 \\
0 & 0 & \sqrt{p_{22}} & 0 \\
0 & 0 & 0 & \sqrt{p_{32}}
\end{array}\right]\left[\begin{array}{l}
s_{1} \\
s_{2} \\
s_{2} \\
s_{3}
\end{array}\right]+\left[\begin{array}{l}
\mathbf{n}_{\mathbf{1}} \\
\mathbf{n}_{\mathbf{2}} \\
\mathbf{n}_{\mathbf{3}}
\end{array}\right]
$$

$$
\text { s.t. }\left\|s_{i}\right\|^{2}=1, \quad\left\|\mathbf{f}_{\mathbf{i}}^{\mathbf{j}}\right\|^{2}=1
$$

\section{TRANSMisSiOn IN THE COOPERATIVE MUlTi-CELL NETWORK}

\section{A. User Equipment Scheduling}

In the cellular wireless communication system, $K$ UEs are distributed randomly in the Rayleigh fading environment. The main goal of this paper is to improve the efficiency of the resource utilization. However, single cell transmission scheme and traditional coordinated multiple cell transmission system need larger transmission power or more system resource. To make use of the resource more efficiently, UE scheduling is inevitable. The multi-cell serves the edge UE jointly. At the same time, each cell also provides the signal transmission for its center UE with better channel quality. A simple user scheduling scheme is used for reducing the complexity of computation. We select the target UE set according to the Frobenius-Norm of the UE's channel matrix in the Rayleigh fading environment. The channel coefficient $\left(h_{p q}\right)$ is independent complex Gaussian random variable with zeros mean and $2 \delta^{2}$ variance $h_{p q} \sim C N\left(0,2 \delta^{2}\right)$. The transmission channel model for UE is defined as Equation (3)

$$
\mathbf{H}=\left[\begin{array}{cccc}
h_{11} & h_{12} & \cdots & h_{1 N_{t}} \\
h_{21} & h_{22} & \cdots & h_{2 N_{t}} \\
\vdots & \vdots & \ddots & \vdots \\
h_{N_{r} 1} & h_{N_{r} 2} & \cdots & h_{N_{r} N_{t}}
\end{array}\right]
$$

And, $\|\mathbf{H}\|_{F}^{2}=\operatorname{trace}\left(\mathbf{H}^{H} \mathbf{H}\right)=\sum_{p=1}^{N_{r}} \sum_{q=1}^{N_{t}}\left|h_{p q}\right|^{2}$ has chi-square distributions with $2 N_{r} N_{t}$ degrees of freedom $\left(x_{2 N_{r} N_{t}}^{2}\right)$. Therefore, the probability density function (pdf) and cumulative density function (cdf) of $\|\mathbf{H}\|_{F}^{2}$ are given by Equation (4).

$$
\begin{aligned}
& p d f \rightarrow f_{\|\mathbf{H}\|_{F}^{2}}(x)=\frac{1}{\delta^{2 N_{r} N_{t}} 2^{N_{r} N_{t}} \Gamma\left(N_{r} N_{t}\right)} x^{N_{r} N_{t}-1} e^{-\frac{x}{2 \delta^{2}}} \\
& c d f \rightarrow F_{\|\mathbf{H}\|_{F}^{2}}(x)=1-e^{-\frac{x}{2 \delta^{2}} \sum_{k=0}^{N_{r} N_{t}-1} \frac{1}{k !}\left(\frac{x}{2 \delta^{2}}\right)^{k}, \quad x \geq 0 ;}
\end{aligned}
$$

According to the characteristic of the distribution, a simple user scheduling algorithm is employed in this paper to select the target UE set which is summarized in Table I.

\section{TABLE I. A LOW COMPLEXITy UsER SCHEDULING AlgORITHM}

$$
\begin{aligned}
& \text { 1. Find an Edge UE for the two fixed cells } \\
& \text { Edge- } \mathrm{UE}=\arg \max _{i \in K}\left(\left\|\mathbf{H}_{\mathrm{i} 1}\right\|_{\mathrm{F}}^{2}+\left\|\mathbf{H}_{\mathrm{i} 2}\right\|_{\mathrm{F}}^{2}\right) \\
& \text { s.t. }\left\|\mathbf{H}_{\mathrm{i} 1}\right\|_{\mathrm{F}}^{2}<\|\mathrm{H}\|_{\mathrm{Threshold}}^{2} \&\left\|\mathbf{H}_{\mathrm{i} 2}\right\|_{\mathrm{F}}^{2}<\|\mathbf{H}\|_{\text {Threshold }}^{2}
\end{aligned}
$$

\section{Find another Center UE for each fixed cell}

For the $j$ th cell: Center-UE $=\arg \max _{k \in K, k \neq i}\left\|\mathbf{H}_{\mathrm{kj}}\right\|_{\mathrm{F}}^{2}$ s.t. $\left\|\mathbf{H}_{\mathrm{kj}}\right\|_{\mathrm{F}}^{2}>\|\mathbf{H}\|_{\text {Threshold }}^{2}, \quad j \in\{1,2\}$

\section{The target $U E$ set as follows:}

Target UE set $=\{$ Center-UE1, Edge-UE2, Center-UE3 $\}$; 


\section{B. Non-coherent Coordinated Transmission/Reception}

In this sub-section, we analyze the signal transmission and reception process for the target UE set which is selected according to the user scheduling algorithm. Our main goal is to improve the sum-rate of the system on the condition of the optimal performance for the edge UE.

To achieve the maximum received SNR for the UE, many interference suppressing technologies can be used, such as transmit and receive precoding. Maximizing the signalinterference-noise-ratio is often used as the criterion. Actually, the interference made by the $i$ th UE can be viewed as the leaked power from this UE to all other UEs. For the $i$ th UE, the effective signal power is $E_{i}^{e}$, while the equivalent sumpower of the leakage and the noise is $E_{i}^{l}$. Therefore, we can get the precoding vector for each UE according to the maximumsignal-leakage-noise-ratio, such that

$$
\begin{gathered}
\arg \max _{\mathbf{f}} \operatorname{SLNR}_{i}=\frac{E_{i}^{e}}{E_{i}^{l}} \\
\operatorname{SLNR}_{1}=\frac{p_{11} s_{1}^{H}\left(\mathbf{H}_{11} \mathbf{f}_{1}^{1}\right)^{H} \mathbf{H}_{11} \mathbf{f}_{1}^{1} s_{1}}{p_{11} s_{1}^{H}\left(\mathbf{H}_{11} \mathbf{f}_{1}^{1}\right)^{H} \mathbf{H}_{21} \mathbf{f}_{1}^{1} s_{1}+\mathbf{n}_{1}^{H} \mathbf{n}_{1}} \\
=\frac{\left(\mathbf{f}_{1}^{1}\right)^{H} p_{11} \mathbf{H}_{11}^{H} \mathbf{H}_{11} \mathbf{f}_{1}^{1}}{\left(\mathbf{f}_{1}^{1}\right)^{H}\left(p_{11} \mathbf{H}_{21}^{H} \mathbf{H}_{21}+\mathbf{n}_{1}^{H} \mathbf{n}_{1} \mathbf{I}_{\mathbf{N}_{\mathbf{t}}}\right) \mathbf{f}_{1}^{1}}
\end{gathered}
$$

From Equation (6), we can see that the generalized eigenvector with the maximum generalized eigenvalue of the matrix pair $\left(p_{11} \mathbf{H}_{21}^{H} \mathbf{H}_{21}+\mathbf{n}_{1}^{H} \mathbf{n}_{1} \mathbf{I}_{\mathbf{N}_{\mathrm{t}}}, p_{11} \mathbf{H}_{11}^{H} \mathbf{H}_{11}\right)$ is the desired precoding vector for the center UE1. Using the same method, transmission precoding vector at each cell for each UE can be achieved as illustrated by Equation (7).

$$
\left\{\begin{array}{l}
\mathbf{f}_{1}^{1} \rightarrow \text { max .eigenvector }\left(\left(p_{11} \mathbf{H}_{21}^{H} \mathbf{H}_{21}+\mathbf{n}_{1}^{H} \mathbf{n}_{1} \mathbf{I}_{\mathbf{N}_{\mathbf{t}}}\right)^{-1} p_{11} \mathbf{H}_{11}^{H} \mathbf{H}_{11}\right) \\
\mathbf{f}_{2}^{1} \rightarrow \max \text {.eigenvector }\left(\left(p_{21} \mathbf{H}_{11}^{H} \mathbf{H}_{11}+\mathbf{n}_{2}^{H} \mathbf{n}_{2} \mathbf{I}_{\mathbf{N}_{\mathbf{t}}}\right)^{-1} p_{21} \mathbf{H}_{21}^{H} \mathbf{H}_{21}\right) \\
\mathbf{f}_{2}^{2} \rightarrow \max \text {.eigenvector }\left(\left(p_{22} \mathbf{H}_{32}^{H} \mathbf{H}_{32}+\mathbf{n}_{2}^{H} \mathbf{n}_{2} \mathbf{I}_{\mathbf{N}_{\mathbf{t}}}\right)^{-1} p_{22} \mathbf{H}_{22}^{H} \mathbf{H}_{22}\right) \\
\mathbf{f}_{3}^{2} \rightarrow \max \text {.eigenvector }\left(\left(p_{32} \mathbf{H}_{22}^{H} \mathbf{H}_{22}+\mathbf{n}_{3}^{H} \mathbf{n}_{3} \mathbf{I}_{\mathbf{N}_{\mathbf{t}}}\right)^{-1} p_{32} \mathbf{H}_{32}^{H} \mathbf{H}_{32}\right)
\end{array}\right.
$$

\section{Power Allocation and System Rate Analysis}

In this sub-section, we focus on the case where the rate of the edge UE is maximized with the total transmitted power constrains. After the maximal-ratio-combining (MRC), we can evaluate the desired signal for each UE as shown in Equation (8). The detection vector $\mathbf{R}$ and the received signalinterference-noise-ratio of the UE are summarized in Table II.

$$
\left[\begin{array}{l}
\hat{s}_{1} \\
\hat{s}_{2} \\
\hat{s}_{3}
\end{array}\right]=\left[\begin{array}{l}
\mathbf{R}_{1} \mathbf{y}_{1} \\
\mathbf{R}_{2} \mathbf{y}_{2} \\
\mathbf{R}_{3} \mathbf{y}_{3}
\end{array}\right]
$$

TABLE II. USER MRC VECTOR AND SINR

\begin{tabular}{|c|c|c|}
\hline $\boldsymbol{U E}$ & MRC Vector (R) & SINR \\
\hline $\mathbf{1}$ & $\frac{\left(\mathbf{H}_{11} \mathbf{f}_{1}^{1}\right)^{H}}{\left\|\mathbf{H}_{11} \mathbf{f}_{1}^{1}\right\|^{2} \sqrt{p_{11}}}$ & Equation (9) \\
\hline $\mathbf{2}$ & $\frac{\left(\mathbf{H}_{21} \mathbf{f}_{2}^{1} \sqrt{p_{21}}+\mathbf{H}_{22} \mathbf{f}_{2}^{2} \sqrt{p_{22}}\right)^{H}}{\left\|\mathbf{H}_{21} \mathbf{f}_{2}^{1} \sqrt{p_{21}}+\mathbf{H}_{22} \mathbf{f}_{2}^{2} \sqrt{p_{22}}\right\|^{2}}$ & Equation (10) \\
\hline $\mathbf{3}$ & $\frac{\left(\mathbf{H}_{32} \mathbf{f}_{3}^{2}\right)^{H}}{\left\|\mathbf{H}_{32} \mathbf{f}_{3}^{2}\right\|^{2} \sqrt{p_{32}}}$ & Equation (11) \\
& & \\
\hline
\end{tabular}

$$
\begin{aligned}
& \operatorname{SINR}_{1}=\frac{p_{11}\left\|\mathbf{H}_{\mathbf{1 1}} \mathbf{f}_{\mathbf{1}}^{1}\right\|^{4}}{p_{21}\left\|\left(\mathbf{H}_{\mathbf{1 1}} \mathbf{f}_{\mathbf{1}}^{\mathbf{1}}\right)^{H} \mathbf{H}_{\mathbf{1 1}} \mathbf{f}_{\mathbf{2}}^{\mathbf{1}}\right\|^{2}+\left\|\left(\mathbf{H}_{\mathbf{1 1}} \mathbf{f}_{\mathbf{1}}^{\mathbf{1}}\right)^{H} \mathbf{n}_{1}\right\|^{2}} \\
& \operatorname{SINR}_{3}=\frac{p_{32}\left\|\mathbf{H}_{\mathbf{3 2}} \mathbf{f}_{\mathbf{3}}^{\mathbf{2}}\right\|^{4}}{p_{22}\left\|\left(\mathbf{H}_{\mathbf{3 2}} \mathbf{f}_{\mathbf{3}}^{\mathbf{2}}\right)^{H} \mathbf{H}_{\mathbf{3 2}} \mathbf{f}_{\mathbf{2}}^{\mathbf{2}}\right\|^{2}+\left\|\left(\mathbf{H}_{\mathbf{3 2}} \mathbf{f}_{\mathbf{3}}^{\mathbf{2}}\right)^{H} \mathbf{n}_{3}\right\|^{2}}
\end{aligned}
$$

According to the Shannon Formula, the system sum-rate and the transmission rate of the edge UE can be calculated by Equation (12) and (13) respectively.

$$
\begin{gathered}
\text { SumRate }=\sum_{i=1}^{3} \log _{2}\left(1+\operatorname{SINR}_{i}\right) \\
\arg \max _{p_{11}, p_{21}, p_{22}, p_{32}} \text { EdgeRate }=\log _{2}\left(1+\operatorname{SINR}_{2}\right)
\end{gathered}
$$

$$
\text { s.t. } p_{11}, p_{21}, p_{22}, p_{32} \in(0,1) \cap p_{11}+p_{21}+p_{22}+p_{32}=1
$$

Now we are going to solve the optimization problem in Equation (13) under the total transmitting power constraints illustrated by Equation (14). For cutting down the computation complexity in Equation (13), firstly, we allocate the total transmission power to the three of UE by Equation (15). Secondly, allocate the power $\left(\sin ^{2} \alpha\right)$ of the edge UE2 to the two of transmitting cell by full researching method. Then, the transmit power of the two cells is $p_{11}+p_{21}$ and $p_{22}+p_{32}$ respectively.

$$
\left\{\begin{array}{l}
p_{11}=\cos ^{2} \alpha \cos ^{2} \beta \\
p_{21}+p_{22}=\sin ^{2} \alpha, \quad \alpha, \beta \in\left(0, \frac{\pi}{2}\right) \\
p_{32}=\cos ^{2} \alpha \sin ^{2} \beta
\end{array}\right.
$$

After the transformation formula, the researching number will be reduced significantly. And, the power allocation will be performed by Equation (16).

$$
\arg \max _{\alpha, \beta} \text { EdgeRate }=\log _{2}\left(1+\operatorname{SINR}_{2}\right)
$$




$$
\begin{array}{r}
\operatorname{SINR}_{2}=\left\|\mathbf{H}_{\mathbf{2 1}} \mathbf{f}_{\mathbf{2}}^{\mathbf{1}} \sqrt{p_{21}}+\mathbf{H}_{\mathbf{2 2}} \mathbf{f}_{\mathbf{2}}^{\mathbf{2}} \sqrt{p_{22}}\right\|^{4} /\left(\left\|\left(\mathbf{H}_{\mathbf{2 1}} \mathbf{f}_{\mathbf{2}}^{\mathbf{1}} \sqrt{p_{21}}+\mathbf{H}_{\mathbf{2 2}} \mathbf{f}_{\mathbf{2}}^{\mathbf{2}} \sqrt{p_{22}}\right)^{H} \mathbf{H}_{\mathbf{2 1}} \mathbf{f}_{\mathbf{1}}^{\mathbf{1}}\right\|^{2} p_{11}+\cdots\right. \\
\left\|\left(\mathbf{H}_{\mathbf{2 1}} \mathbf{f}_{2}^{\mathbf{1}} \sqrt{p_{21}}+\mathbf{H}_{\mathbf{2 2}} \mathbf{f}_{\mathbf{2}}^{\mathbf{2}} \sqrt{p_{22}}\right)^{H} \mathbf{H}_{\mathbf{2 2}} \mathbf{f}_{\mathbf{3}}^{\mathbf{2}}\right\|^{2} p_{32}+\cdots \\
\left.\left\|\left(\mathbf{H}_{\mathbf{2 1}} \mathbf{f}_{\mathbf{2}}^{\mathbf{1}} \sqrt{p_{21}}+\mathbf{H}_{\mathbf{2 2}} \mathbf{f}_{\mathbf{2}}^{\mathbf{2}} \sqrt{p_{22}}\right)^{H} \mathbf{n}_{2}\right\|^{2}\right)
\end{array}
$$

\section{NUMERICAL SIMULATION}

Besides perfect CSI at the transmitter, we assume that the signal-noise-ratio at the Center UE is about $13 \mathrm{~dB}$ larger than that of the Edge UE. In our simulation, to make a more real environment, we set the SNR of the edge UE from $-10 \mathrm{~dB}$ to $2 \mathrm{~dB}$, and the SNR of the center UE from $3 \mathrm{~dB}$ to $15 \mathrm{~dB}$, and the total number of UE is set as $K=30$ in the system. In this work, we just consider the single stream transmission for each UE. The QPSK constellation is employed as the modulation method in all simulations. Monte Carlo simulations for each SNR value were used to obtain the results. And, it is noted that the SNR values in the X-axis denotes the SNR of the edge UE in our all simulation results.

Assume the quasi-static channel model with its elements generated as zero-mean and unit-variance $h_{p q} \sim C N(0,1)$ independent and identically distributed (i.i.d) complex Gaussian random variables in the simulation. In Fig. 2, we compare the derived theoretical CDF of $\|\mathbf{H}\|_{\mathrm{F}}^{2}$ with the empirical CDF generated by many channel realizations for different $N_{t}$ and $N_{r}$. In the simulation, we generate $10^{6}$ random channels realization to validate the derived CDF with the empirical CDF. An appropriate $\|\mathbf{H}\|^{2}$ Threshold for differentiating the edge UE and the center UE can be set. We confirm the $\|\mathbf{H}\|^{2}{ }_{\text {Threshold }}$ when the cumulative density is equal to $5 \%$ for maintaining the probability of the edge UE is the $5 \%$ under the system configuration with $N_{t}=4, N_{r}=2$. The results shown in Fig. 2 prove that our user scheduling algorithm is available.

We also compare the rate performance with the different transmission system for serving the edge UE. The traditional single cell transmission for single edge UE (Single-cell+SUMIMO) and the cooperative multi-cell transmission for single edge UE (Multi-cell+SU-MIMO) with equipped multiple antennas both at the transmitter and receiver $\left(N_{t}=4, N_{r}=2\right)$ are selected as the benchmark. These two benchmarks have the same signal-processing flows with the proposed scheme (Proposed+MU-MIMO). In fact, the edge UE often has very low received SNR due to long distance with the transmitter. At the same time, the channel matrix square norm of the edge UE is also very low under the flat Rayleigh fading environment according to the analysis above. Therefore, the system resource still has large waste if providing service for only one edge UE. However, this work proposes a cooperative multicell transmission scheme for multi-user system, and the total transmission power is constrained with simple user scheduling as shown in Table I, as well as power allocation algorithm to overcome the limitation. Fig. 3 illustrates the proposed scheme

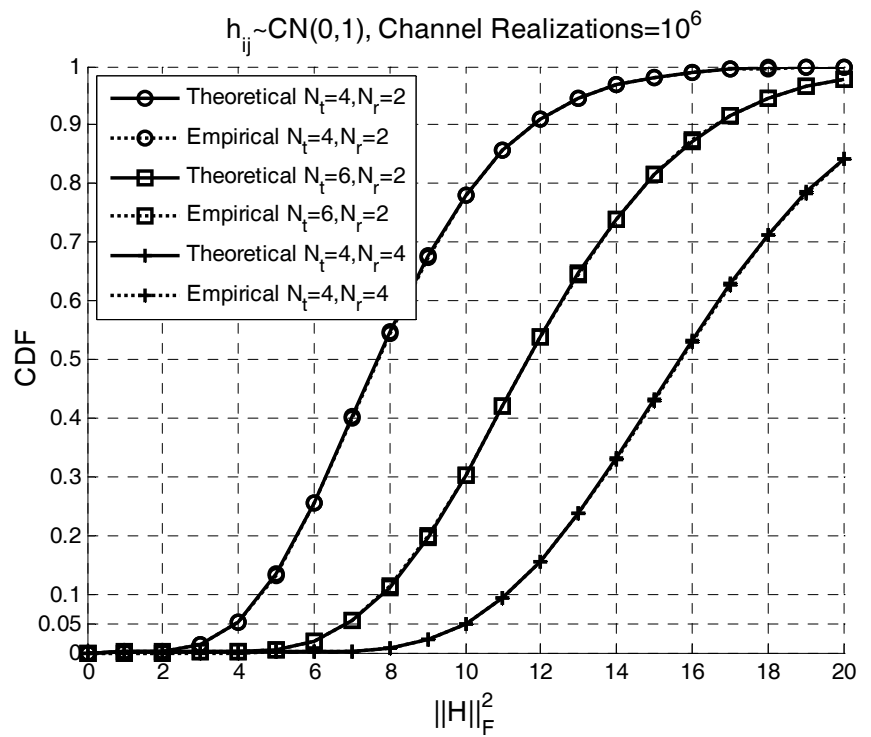

Figure 2. Cumulative density distribution of $\|\mathrm{H}\|_{\mathrm{F}}^{2}$ in the flat Rayleigh fading environment

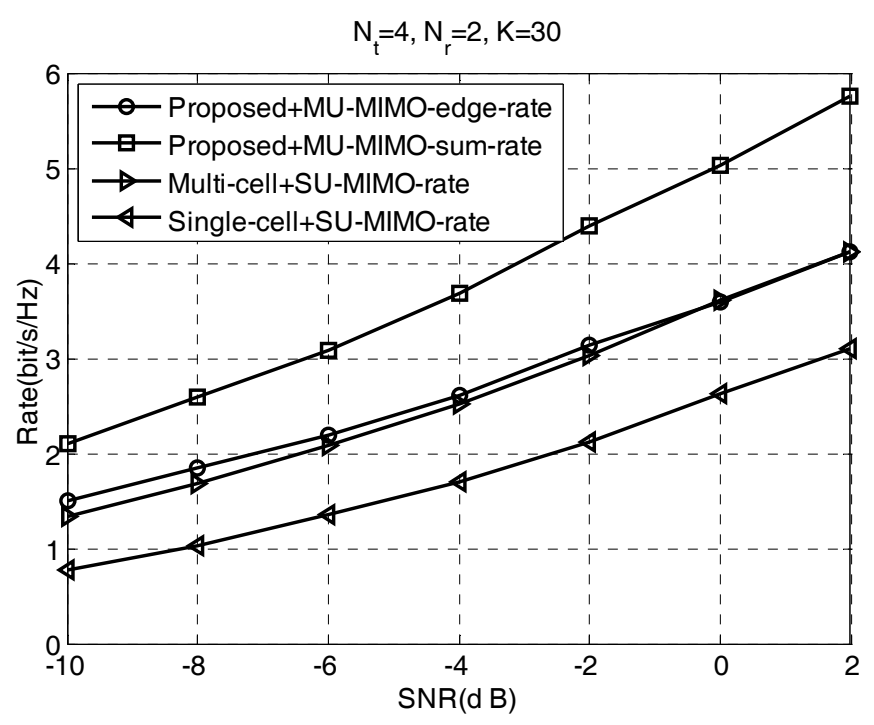

Figure 3. Rate performance of the system, when $\mathrm{N}_{\mathrm{t}}=4, \mathrm{~N}_{\mathrm{r}}=2, \mathrm{~K}=30$

which can improve both the sum-rate of the system and the rate of the edge UE compared with the benchmarks.

Additionally, Fig. 4 compares the raw BER performance of the proposed scheme with the traditional transmission schemes 


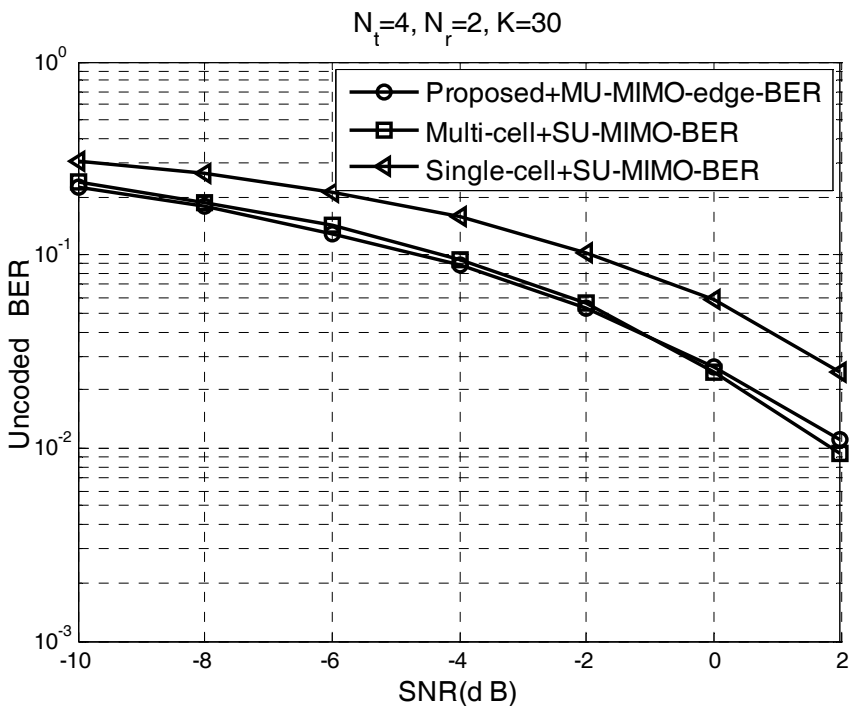

Figure 4. Raw-BER performance of the system, when $\mathrm{N}_{\mathrm{t}}=4, \mathrm{~N}_{\mathrm{r}}=2, \mathrm{~K}=30$

(Single-cell+SU-MIMO and Multi-cell+SU-MIMO). Due to the effective interference suppressing technology and optimal power searching scheme, the proposed transmission can suppress the interference which is made by the other two center UEs for the edge UE. The results verify that our transmission scheme also maintains the raw BER performance of the edge UE.

\section{CONCLUSION}

In this work, an efficient downlink cooperative multi-cell transmission multiple-input-multiple-output system for multiuser was investigated. To enable practical implementation, a simple user scheduling algorithm and a power allocation scheme are proposed to solve the problem of resource waste in the traditional single user system. Moreover, the proposed transmission scheme can obtain the additional multi-user diversity gain so that it can effectively improve the system performance. According to the theoretical analysis and simulation, for one thing, the results confirm that the system transmission sum-rate achieves the improvement due to designing the transmission scheme as a whole. For another thing, the performance of the edge UE also has got improved. At the same time, the low complexity of the proposed scheme is maintained.

\section{REFERENCES}

[1] H. Weigarten, Y. Steinberg, and S. Shamai (Shitz), "The capacity region of the Gaussian multiple-input multiple-output broadcast channel," IEEE Trans. Info. Th., vol. 52, Page(s):3936-3964, Sep. 2006.

[2] Sadek, M., Tarighat, A., and Sayed, A.H., "A Leakage-Based Precoding Scheme for Downlink Multi-User MIMO Channels," IEEE Transactions on Wireless Communications, Volume 6, Issue 5, May 2007, Page(s):1711-1721.

[3] Q. H. Spencer, A. L. Swindlehurst, and M. Haardt, "Zero-forcing methods for downlink spatial multiplexing in multiuser MIMO channels," IEEE Transactions on Signal Processing, vol. 52, Page(s):461-471, Feb. 2004.

[4] R. Chen, J. G. Andrews, and R. W. Heath, "Multiuser space-time block coded MIMO system with unitary downlink precoding," in Proc. IEEE
International Conference on Communications, Paris, France, June 2004, Page(s):2689-2693.

[5] L. Choi, and R. D. Murch, "A transmit preprocessing technique for multiuser MIMO systems using a decomposition approach," IEEE Trans. Wireless Comm., vol. 52, Page(s):773 -786, July 2003.

[6] Taesang Yoo, and Goldsmith, A., "Optimality of zero-forcing beamforming with multiuser diversity," IEEE International Conference on Communications, 2005. Volume 1, 16-20 May 2005 Page(s):542546.

[7] Shamai, S., and Zaidel, B.M., "Enhancing the cellular downlink capacity via co-processing at the transmitting end," IEEE VTS 53rd, Vehicular Technology Conference, 2001. Volume 3, 6-9 May 2001 Page(s): $1745-1749$.

[8] O. Somekh, B. M. Zaidel, and S. Shamai (Shitz), "Sumrate characterization of joint multiple cell-site processing," in Proceedings of the 9th Canadian Workshop on Information Theory (CWIT '05), Montreal, Canada, June 2005

[9] Tamaki, T., Kibeom Seong, and Cioffi, J.M., "Downlink MIMO Systems Using Cooperation Among Base Stations in a Slow Fading Channel," IEEE International Conference on Communications, 2007. 24-28 June 2007 Page(s):4728 - 4733.

[10] Somekh, O., Simeone, O., Bar-Ness, Y., and Haimovich, A.M., "Distributed Multi-Cell Zero-Forcing Beamforming in Cellular Downlink Channels," Global Telecommunications Conference, 2006. Nov. 27 2006-Dec. 12006 Page(s): 1 - 6.

[11] H. Zhang, H. Dai, and Q. Zhou, "Base station cooperation for multiuser MIMO: Joint transmission and BS selection," in Proceedings of the 2004 Conference on Information Sciences and Systems (CISS'04), (Princeton University, Princeton, NJ), Mar. 17 - 19.

[12] Dahrouj, H., and Wei Yu, "Coordinated beamforming for the multi-cell multi-antenna wireless system," 42nd Annual Conference on Information Sciences and Systems, 2008. 19-21 March 2008 Page(s):429 - 434.

[13] Antti Tolli, Marian Codreanu, and Markku Juntti, "Linear cooperative multiuser MIMO transceiver design with per BS power constraints," IEEE International Conference on Communications, 2007. 24-28 June 2007 Page(s):4991 - 4996. 